\title{
Dynamics of 1-D Electron Motion under a Periodic Electric Field with Radiation Reaction Effect
}

\author{
Gustavo V. López, Jorge Lizarraga \\ Departamento de Física, Universidad de Guadalajara, Blvd. Marcelino García Barragan y Calzada Olímpica, Guadalajara, Jalisco, \\ Mexico \\ Email: gulopez@cencar.udg.mx, thejorge@live.com.mx
}

How to cite this paper: López, G.V. and Lizarraga, J. (2020) Dynamics of 1-D Electron Motion under a Periodic Electric Field with Radiation Reaction Effect. Journal of Applied Mathematics and Physics, 8, 814-820. https://doi.org/10.4236/jamp.2020.85063

Received: March 28, 2020

Accepted: May 6, 2020

Published: May 9, 2020

Copyright () 2020 by author(s) and Scientific Research Publishing Inc. This work is licensed under the Creative Commons Attribution International License (CC BY 4.0).

http://creativecommons.org/licenses/by/4.0/

\section{(c) (i) Open Access}

\begin{abstract}
We consider the 1-D motion of an electron under a periodic force and take into account the effect of radiation reaction dissipation force on its motion, using the formulation of novel radiation reaction force as a function of the external force expression. Two cases are considered: a simple sinusoidal time depending force, and sinusoidal electromagnetic force with position and time dependence. The purpose of the study of these cases is to bring about a way to find an experimental study to determine the validity of this novel approach for the radiation reaction force. We found that the difference of the normalized (with respect the speed of light) velocities, with and without radiation reaction, is quite small between $10^{-31}$ to $10^{-14}$ for intensities on the electric field of $10^{-8}$ to 1 Dynes/ues, which may represent some concern to measure experimentally.
\end{abstract}

\section{Keywords}

Radiation Reaction Force, 1-D Electron Motion, Damping Motion

\section{Introduction}

Emission of electromagnetic radiation is a natural classical phenomenon appearing because of the acceleration of any charged particle [1] [2]. This radiation implies emission of energy of the charge and, consequently, a damping effect on its motion, which is modeled through a force called "radiation reaction force". An alternative formulation to the usual Abraham-Lorentz-Dirac radiation reaction force [3] [4] [5] has appeared recently [6] [7], where the radiation reaction force is given in terms of the same external force which brings about the accele- 
ration of the charge. This approach intents to solve some difficulties of the previous formulation [8]-[15], where the radiation reaction is given in terms of the acceleration which brings about pre and post acceleration of a charged particle after the external force appears and disappears, having no experimental or consistent theoretical justification. The novel approach to radiation reaction has not been experimentally tested yet, although some theoretical studies have been done already [6] [16]. Because of this experimental requirement, we make in this paper a study of the 1-D motion of an electron under a periodic external force. The modified relativistic equation of 1-D motion of a charged particle is given by [6]

$$
\frac{\mathrm{d}(\gamma m v)}{\mathrm{d} t}=F-\frac{\lambda_{0} F^{2}}{v}, \quad \lambda_{0}=\frac{2 q^{2}}{3 m^{2} c^{3}} .
$$

where $\gamma=\left(1-\beta^{2}\right)^{-1 / 2}$ with $\beta=v / c$ being the normalized velocity of the charge, and $F$ is the external force. This is the equation of motion that we will use in our study. We will study first the dynamics under the simple sinusoidal force

$$
F=q E_{0} \cos (\omega t+\phi),
$$

where $E_{0}, \omega$ and $\phi$ are the amplitude, the angular frequency and the phases of the electric field, and then we will extend our study to the position and time depending force

$$
F=q E_{0} \cos (k x-\omega t+\phi), \quad k=\omega / c .
$$

\section{Dynamical Equations}

Using the external force (2) and (3) in (1), and after some rearrangements, we get the following dynamical systems

$$
\begin{gathered}
\dot{\beta}=\left(q E_{0} \cos (\omega t+\phi)-\frac{q^{2} \lambda_{0} E_{0}^{2}}{\beta c} \cos ^{2}(\omega t+\phi)\right) \frac{\left(1-\beta^{2}\right)^{3 / 2}}{m c} \\
\dot{x}=\beta c
\end{gathered}
$$

and

$$
\begin{gathered}
\dot{\beta}=\left(q E_{0} \cos (k x-\omega t+\phi)-\frac{q^{2} \lambda_{0} E_{0}^{2}}{\beta c} \cos ^{2}(k x-\omega t+\phi)\right) \frac{\left(1-\beta^{2}\right)^{3 / 2}}{m c} \\
\dot{x}=\beta c,
\end{gathered}
$$

where the term $\left(1-\beta^{2}\right)^{3 / 2}$ in (4a) and (5a) appears from the differentiation of $\gamma$ with respect the time. In the first dynamical system the motion in the coordinates " $x$ " and " $\beta$ " are disconnected, but on the second dynamical system these coordinates are coupled. These dynamical systems are solved using Runge-Kutta method at fourth order and are solved for the cases without radiation reaction force $\left(\lambda_{0}=0\right.$, the solution is denoted as $\left.\beta_{0}(t)\right)$ and with radiation reaction force $\left(\lambda_{0} \neq 0\right.$, the solution is denoted as $\left.\beta(t)\right)$. Then, we calculate the 
difference

$$
\Delta \beta=\beta(t)-\beta_{0}(t) .
$$

\section{Numerical Analysis}

We solved the first dynamical system, Equations (4a) and (4b), for different but fixed $E_{0}$ (electric field intensities) to see the change in the electron's dynamics behavior. This is done for $\lambda_{0}=0,\left(x_{0}(t), \beta_{0}(t)\right)$, and for $\lambda_{0} \neq 0,(x(t), \beta(t))$, simultaneously at each time " $t$ ", and the difference (6) is calculated. The inital conditions are the same, position $x_{0}(0)=x(0)$ and normalized velocity $\beta_{0}(0)=\beta(0)$. Figure 1 shows the expression $\ln |\Delta \beta|$, solution of first dynamical system with Equations (4a) and (4b), as a function of time for several $E_{0}$ (Dynes/ues) values. As one can see, this figure has some peaks and discontinuity, the one that is in the middle can be associated with a change of force's direction, this will happen when $\omega t=\pi$ (for this case $\omega=10^{9} \mathrm{~Hz}$ and $\phi=0$ ). The peaks at the very beginning are due to the fact that $\beta$ and $\beta_{0}$ are very close together in magnitude, that is $\Delta \beta \sim 0$ and as is known $\ln (0)=-\infty$. In addition, we can see that the difference $\Delta \beta$ increases with the intensity of electric field. From Equation (4a) we notice that when the electron's normalized velocity is closed to one ( $\beta \sim 1$ ), the external force approaches to zero leading to a $\Delta \beta$ almost constant, and this is observed for $E_{0}=1$. As stronger the electric field

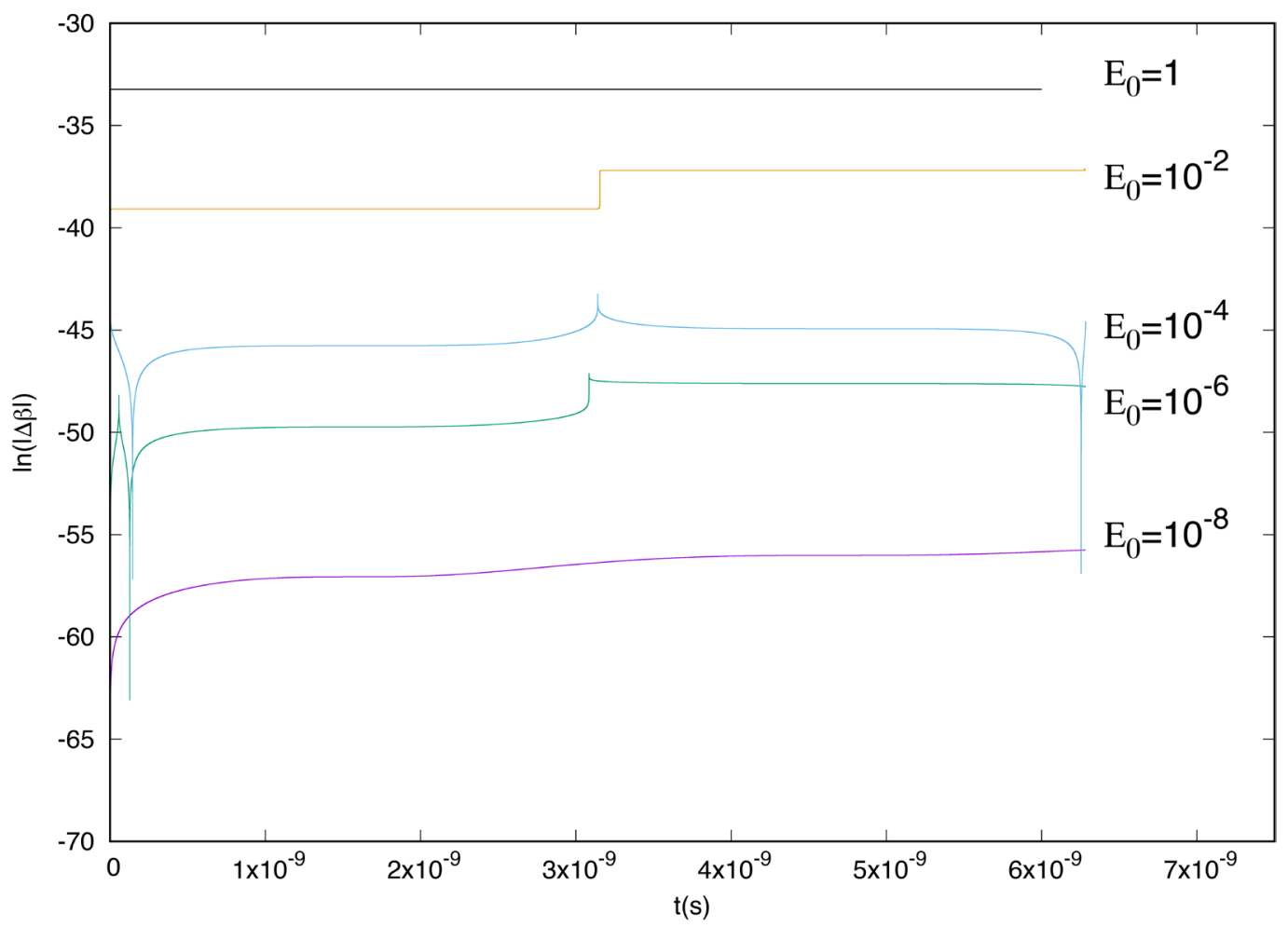

Figure 1. Dynamical system (4): Logarithm of $|\Delta \beta|$ as a function of time for different electric field intensities in units of Dynes/ues with a fixed frequency of $\omega=10 \mathrm{GHz}$. Each curve is associated to the shown intensity of the electric field $E_{0}$. 
is, the difference between non-radiation reaction dynamics and with radiation reaction dynamics become more separated.

Figure 2 shows the same function as before but with the solutions for the second dynamical system (5a) and (5b). For this case, the electron's behavior is a little more complicated, as we can see there are more peaks and discontinuities. As $E_{0}$ increases the difference $\Delta \beta$ increases for times above $3 \times 10^{-7} \mathrm{sec}$ where almost a constant value is reached. The peaks appear because the difference between normalized velocities starts to decrease at those points, and this occurs when the $\beta_{0}$ is very close to the speed of light. Thus from (5a), the external force starts to decrease leading to $\Delta \beta \sim 0$.

Finally we studied the case for different frequencies leaving the electric field intensity fixed at $E_{0}=1$ Dynes/ues. The results for the dynamical system (4a) and (4b) are shown in Figure 3. We can see that for higher frequencies it appears more peaks and discontinuities at the beginning of the motion of the electron, and the reason is due to the same as the previous case, electron speed leads to a difference $\Delta \beta \sim 0$. Also we have three cases of interest here, first one for frequencies of order $1 \mathrm{GHz}$ to $10 \mathrm{GHz}$, the second one for $100 \mathrm{GHz}$ to $1000 \mathrm{GHz}$ and the last one when $\omega \sim 10000 \mathrm{GHz}$. For the first case, we observe that $\Delta \beta$ is almost constant, this is because electron's speed reaches out to the relativistic case very soon and the external force disappears. For the second one, force's frequency leads to a change of force's direction in a way that the electron doesn't

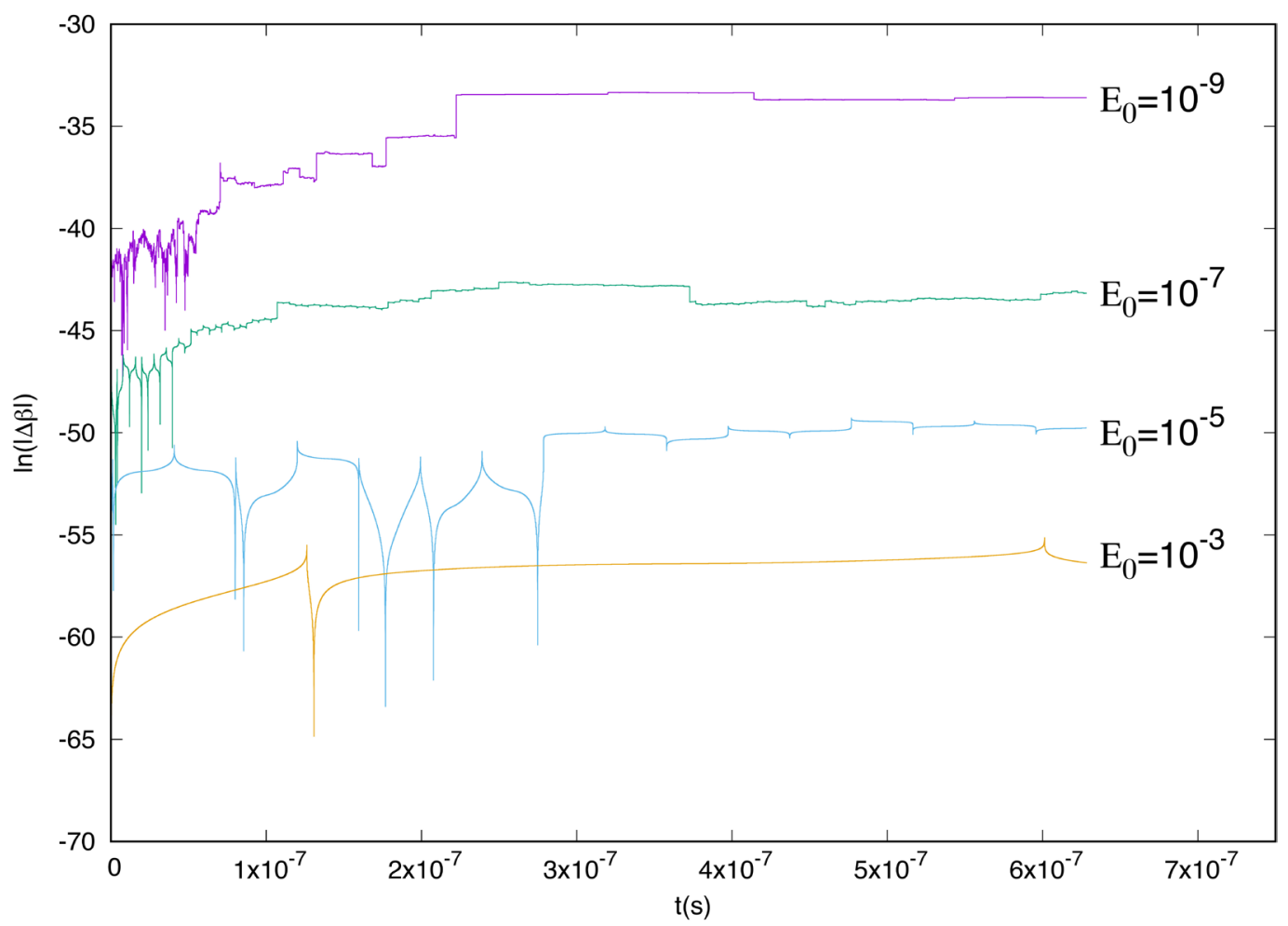

Figure 2. Dynamical system (5): Logarithm of $|\Delta \beta|$ as a function of time for fixed frequency $\omega=10 \mathrm{GHz}$. Each curve is associated to the shown intensity of the electric field $E_{0}$. 


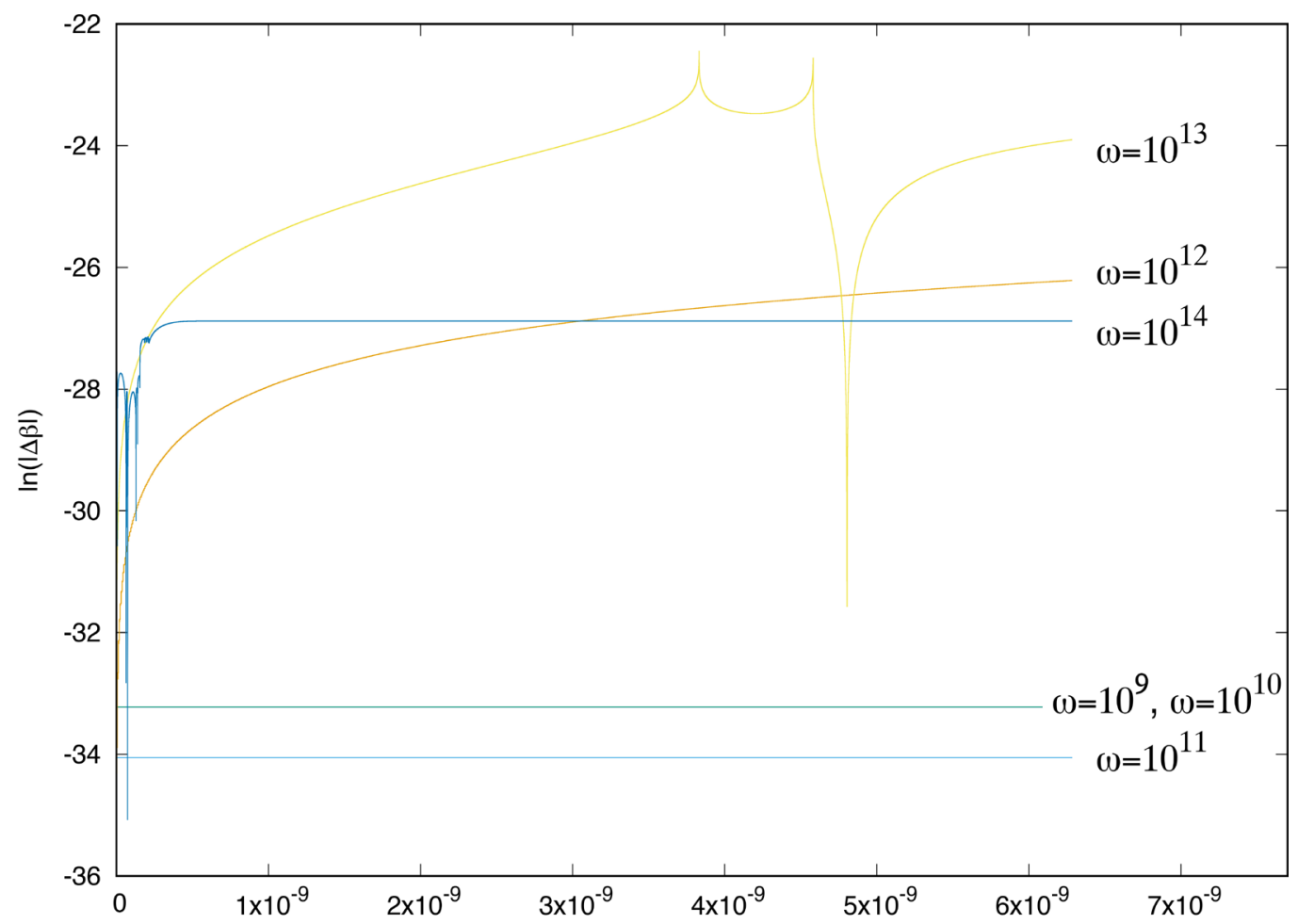

$t(s)$

Figure 3. Dynamical system (4): Logarithm of $|\Delta \beta|$ as a function of time for different frequencies (in $\mathrm{Hz}$ ) and fixed $E_{0}=1$ Dynes/ues .

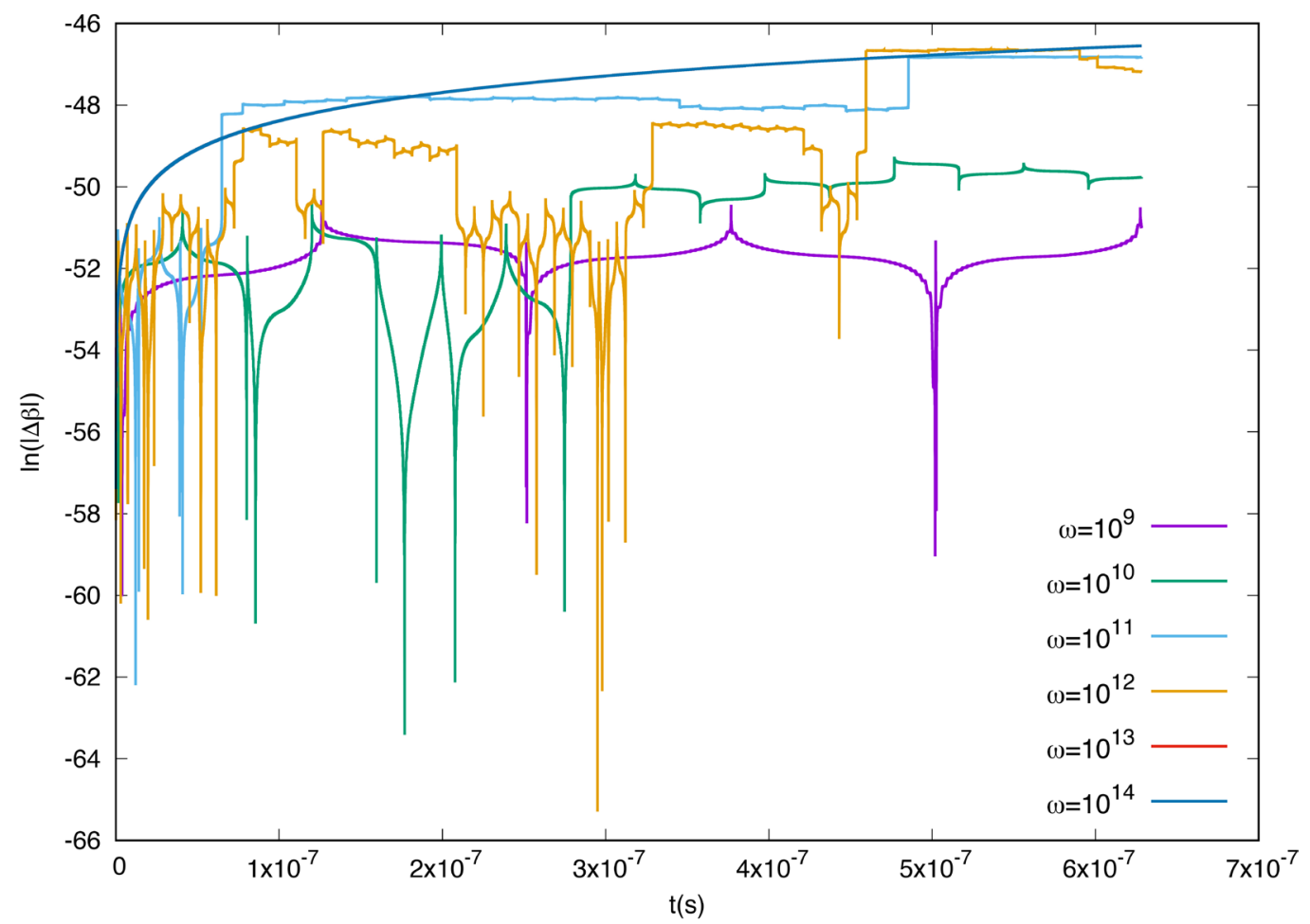

Figure 4. Dynamical system (5): Logarithm of $|\Delta \beta|$ as a function of time for fixed $E_{0}=10^{-7}$ Dynes/ues and several frequencies (in $\mathrm{Hz}$ ). 
reach the relativistic case for the simulation time considered. And for the last case, the frequency makes the force changes direction so rapidly that we start to have a lot of discontinuities until electron speed is stable.

For the dynamical system (5) in Figure 4, we have fixed the electric field intensity at $E_{0}=1 \times 10^{-7}$ Dynes/ues and we change the frequencies from $\omega=(1,1000) \mathrm{GHz}$. One can see that electron's behavior is very complicated, we have more discontinuities while the frequency is higher until the electron reaches a relativistic speed at frequency $\omega=1 \times 1000 \mathrm{GHz}$ and the external force disappears. Those discontinuities can be explained similarly as we have done with all the previous cases.

\section{Conclusion}

We studied 1-D electron motion under two periodic forces and including the novel radiation reaction force. When the periodic external force has a simple time dependence (dynamical system (4)), we found that the difference $\Delta \beta$ increases with $E_{0}$. For the periodic force depending on position and time (dynamical system (5)), the relation between $\Delta \beta$ and $E_{0}$ is more complicated but the same dependence is observed after some time $\left(3 \times 10^{-7} \mathrm{sec}\right)$ when the difference becomes more stable. On the other hand and for both dynamical systems, the relation between the frequency of the electric field and $\Delta \beta$ is different since several peaks and discontinuities appear. The observed value for $\Delta \beta$ for both cases is of the order of $10^{-31}$ to $10^{-14}$ which could be a concern to detect experimentally, but we think it is worthy to try to make an experiment to see whether or not this approach for radiation reaction force points to the right experimental direction.

\section{Conflicts of Interest}

The authors declare no conflicts of interest regarding the publication of this paper.

\section{References}

[1] Jackson, J.D. (1962) Classical Electrodynamics. John Wiley and Sons Inc., Hoboken.

[2] Landau, L.D. and Lifschitz, E.M. (1971) The Classical Theory of Fields. Pergamon Press, Oxford.

[3] Abraham, M. (1905) Theorie de Electrizitat, Volume II. Teubner, Leipzig.

[4] Lorentz, H.A. (1909) The Theory of Electrons. Teubner, Leipzig.

[5] Dirac, P.A.M. (1938) Classical Theory of Radiating Electrons. Proceedings of the Royal Society of London. Series A, Mathematical and Physical Sciences, 167, 148-169. https://doi.org/10.1098/rspa.1938.0124

[6] López, G.V. (2016) Force Approach to Radiation Reaction. Annals of Physics, 365, 1-6. https://doi.org/10.1016/j.aop.2015.11.012

[7] López, G.V. (2017) Generalization of the Force Approach to Radiation Reaction. Journal of Applied Mathematics and Physics, 5, 569.

https://doi.org/10.4236/jamp.2017.53048 
[8] Comay, E. (1993) Remarks on the Physical Meaning of the Lorentz-Dirac Equation. Foundations of Physics, 23, 1121-1136. https://doi.org/10.1007/BF00732416

[9] Valentini, A. (1988) Resolution of Causality Violation in the Classical Radiation Reaction. Physical Review Letters, 61, 1903. https://doi.org/10.1103/PhysRevLett.61.1903

[10] Spohn, H. (2004) Dynamics of Charged Particles and Their Radiation Field. Cambridge University Press, Cambridge. https://doi.org/10.1017/CBO9780511535178

[11] Heras, J.A. (2006) Preacceleration without Radiation: The Nonexistence of Preradiation Phenomenon. American Journal of Physics, 74, 1025-1030. https://doi.org/10.1119/1.2221342

[12] Jackson, J.D. (2007) Comment on "Preacceleration without Radiation: The Nonexistence of Preradiation Phenomenon," by J.A. Heras. American Journal of Physics, 75, 844-845. https://doi.org/10.1119/1.2733681

[13] Hnizdo, V. (2007) Comment on "Preacceleration without Radiation: The Nonexistence of Preradiation Phenomenon," by J.A. Heras [Am. J. Phys. 74(11), 1025-1030 (2006)]. American Journal of Physics, 75, 845-846.

https://doi.org/10.1119/1.2733682

[14] Heras, J.A. (2007) Can Maxwells Equations Be Obtained from the Continuity Equation? American Journal of Physics, 75, 652-657. https://doi.org/10.1119/1.2739570

[15] Griffiths, D.J., Proctor, T.C. and Schroeter, D.F. (2010) Abraham-Lorentz versus Landau-Lifshitz. American Journal of Physics, 78, 391-402.

https://doi.org/10.1119/1.3269900

[16] López, G.V. and Lizarraga, J. (2016) Force-Depending Radiation Reaction Study in an Undulator Device. 